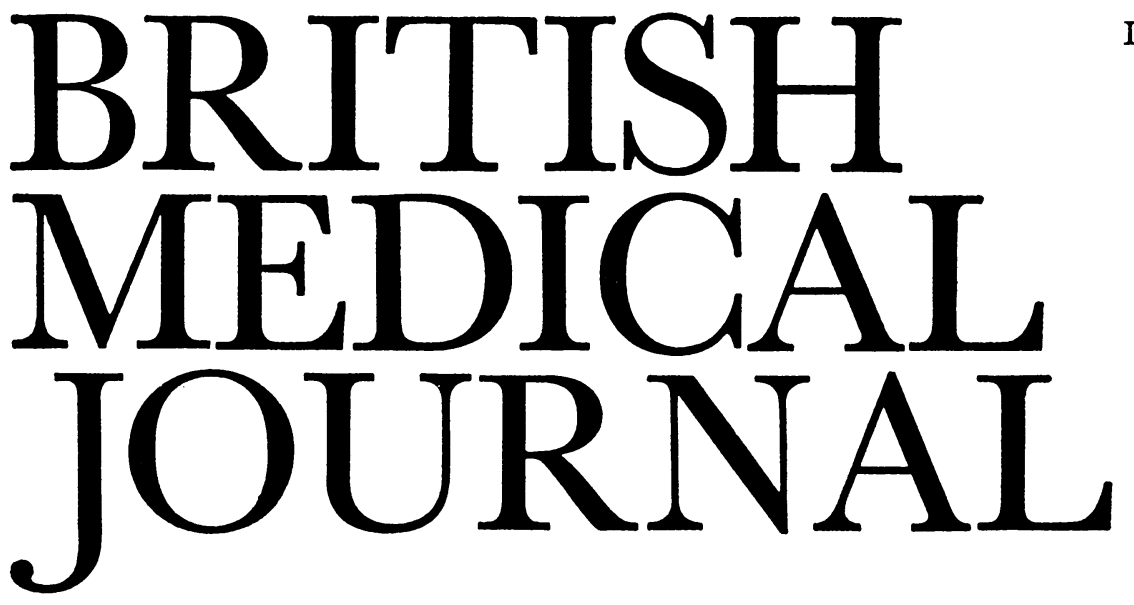

\title{
Examination of the Unconscious Patient
}

Sometimes to be found in hospital case notes is the statement: "Neurological examination impossible because patient unconscious." This is a sad comment on the writer's neurological knowledge, for careful examination of the unconscious patient allows localization of the lesion at least as clearly as in the conscious patient.

The aim should be methodically to assess the integrity of the cerebral hemispheres and brainstem. The degree of impairment of consciousness must first be defined. Coma may result from damage both to the cerebral hemispheres and to the reticular activating system of the brainstem. The parts played by either of these cannot be assessed until the remainder of the neurological examination is completed. Patients may be encountered rarely in whom a brainstem lesion has abolished almost every voluntary movement but leaving the reticular activating system and therefore consciousness unimpaired.

The posture of the patient may be of initial help. The patient may have opisthotonus or neck rigidity from meningitis, or be curled up away from the light from cerebral irritation. Spontaneous or pain-induced decerebrate or decorticate posturing may indicate lesions lying respectively in or above the brainstem. The size of the pupils and their reaction to light will indicate much about the optic nerves, the mid-brain, the third nerve and the sympathetic pathways. The unilateral, dilated, fixed pupil of a third nerve lesion; the bilateral unreactive mediumsize, often irregular pupils of mid-brain lesions; and the pinpoint pupils of pontine lesions may be noted. Bilateral pupillary dilatation on neck flexion suggests severe tentorial herniation.

Lateral deviation of the eyes may result from an ipsilateral destructive lesion or a contralateral irritative lesion of the frontal lobe eyefields, or from a contralateral lateral pontine lesion. Downward deviation of the eyes indicates a lesion in the pretectal area, and skew deviation and dysconjugate eye movements result from intrinsic brainstem disease.

Eye movements may be assessed by means of oculocephalic tests and the response to irrigation of the external auditory canals with cold water. Full range of movement with these procedures indicates integrity of the cranial nerves subserving eye movements and the mid-brain and of the upper cervical spinal cord and the vestibular pathways. Tests of optokinetic nystagmus, which can be used to assess pathways from the eyes through the occipital cortex to the mid-brain, are usually of use only in conscious patients.
The lower cranial nerves can to a greater or less extent be tested directly in unconscious patients. Impairment of the corneal reflex or grimace to pain, including firm supraorbital pressure, on one or other side of the face indicates a lesion of the fifth or seventh nerve or of the adjacent brainstem on that side. The presence of a grimace reaction to pain elsewhere on the body may help in elucidating this. The palatal and gag reflexes may be absent in coma from many causes, but preservation of these reflexes indicates that the ninth and tenth nerves and the lower medullary centres are intact. Abnormalities of respiration or of body temperature are usually of no localizing value, for, though they can arise respectively from brainstem or hypothalamic damage, they are much more commonly due to metabolic disorders. The unilateral dilatation of the pupil in response to pain applied to the side of the neck demonstrates that the spinal cord from about C3 to T1 and sympathetic pathwavs to the eye remain intact on that side.

The well-known signs of an upper motor neuron lesion include increased tendon reflexes and an extensor plantar response on the opposite side of the body from lesions lying above the pyramidal decussation. In addition, a number of primitive reflexes may be uncovered by such lesions. Primitive reflexes are those present in more primitive animals or in infants before maturation of the central nervous system, though some may persist in normal adults to a minimal degree. In the present context, the highest level at which such abnormal reflexes appear indicates the lower limit of destruction brought about by the lesion itself. A palmomental reflex implies an upper motor neuron lesion lying above the seventh nerve nucleus. A snout reflex, utilizing the fifth and seventh nerves, and an increased jaw jerk (being entirely conveved by the fifth nerve) indicate a lesion lying above the fifth nerve nucleus in the pons. A suck reflex and a forced-grasp reflex may not be entirely due to lesions of the upper motor neuron itself, but usually arise from cerebral cortical damage, frequently of the frontal lobes.

R. M. Gordon and M. B. Bender ${ }^{1}$ have recently drawn attention to a relatively rare phenomenon, the corneomandibular reflex. In patients exhibiting this, firm pressure (but not light touch) on one cornea (but not the sclera) causes deviation of the jaw to the opposite side. This is presumably due to activation of the pterygoid muscles on that side. Unilateral wrinkling of the muscles of the chin, the corneomental reflex, was also frequently seen. The pathway for this reflex is through the fifth nerve, and its significance 
is thus exactly the same as that of an increased jaw jerk. They described eight patients seen in two centres in the United States with this reflex. It was particularly distinct in seven, four with cerebral and brainstem vascular disease, one with a cerebral tumour, one with a probable cereb 1 llopontine angle tumour, and one with probable encephalomyelitis. The reflex had the advantage when compared with the jaw jerk in that an increased reflex on one side indicated an upper motor neuron lesion predominating on that side. One patient with amyotrophic lateral sclerosis had a weakly positive corneomandibular reflex. Gordon and Bender attribute the first description of this reflex to von Sölder in $1902^{2}$ and detail the various studies of it from that date. It is clearly rare, for they found it to be present in only one of 300 randomly selected neurological patients, a woman with a thoracic myelopathy due to lymphosarcoma and no clinical evidence of brain disease. On the other hand the corneomental reflex was found in $10-15 \%$ of patients, some with no evidence of brain disease.

Neurosurgeons, particularly those dealing with head injuries, are well versed in the examination outlined here of the patient with disturbed consciousness. On the neurological side, as Gordon and Bender's study indicates, that commonest of neurological conditions, cerebrovascular disease, provides the greatest number of patients with dis- turbed consciousness. Understanding of stupor and coma has reached its peak in the U.S.A., ${ }^{3}$ where neurologists in many centres have for many years concerned themselves with patients with "strokes." In the United Kingdom, unfortunately, neurologists as a whole have found themselves unable to look after the large number of patients with cerebrovascular disease. Consequently the level of special skill, and so of teaching, in this branch of medicine has dropped behind that in America.

The time has come to accept the lesson offered by the transatlantic experience, and to consider the creation of regional "stroke units" along the lines of the recently developed coronary care units. These units would need both to care for the patient in the acute phase of his illness and to have full rehabilitation services, which are so hard pressed in this country at present. A considerable expansion of the number of neurologists and specialists in rehabilitation medicine would also be required. Nevertheless, the long-term benefit in the welfare of patients with strokes and in the teaching of neurology demands that we seriously consider the development of stroke units in this country.

\footnotetext{
Gordon, R. M., and Bender, M. B., fournal of Neurology, Neurosurgery and Psychiatry, 1971, 34, 236.

2 Sölder, F. von, Neurologisches Zentralblatt, 1902, 21, 111.

3 Plum, F., and Posner, J. B., The Diagnosis of Stupor and Coma. Oxford, Blackwell, 1966.
}

\section{Detection of Regional Left Ventricular Dysfunction}

The first 10 years of coronary care have been devoted mainly to the prevention and treatment of dysrhythmias, the common cause of early death after myocardial infarction. Now more attention is paid to conservation of the myocardium, because those patients who are saved from death due to dysrhythmia will eventually succumb to loss of myocardium.

Besides relieving angina, a successful operation of aortocoronary bypass grafting ${ }^{1} 2$ should protect the patient against future infarction not only in the immediate territory of the grafted coronary artery but also in adjacent territories, for collaterals may become available there when atheroma blocks other major coronary vessels. It is now possible to perform revascularization as an urgent operation in patients with preinfarction angina and to consider revascularization combined with excision of the infarct for the critically ill patient who is otherwise unlikely to survive. ${ }^{3}$ Surgical removal of the hindrance to proper contraction of the heart owing to a dead segment of left ventricular wall is only beginning to find a place in the treatment of the acute phase of infarction.

Non-elective surgical excision of a chronic left ventricular aneurysm or of damaged myocardium has been successfully practised for nearly a decade, ${ }^{4}$ but improvements in diagnostic techniques and in knowledge of the effect of aneurysm on total left ventricular function have greatly aided the selection of patients. Consequently more patients are now held to be suitable for aneurysmectomy, and this can often profitably be combined with revascularization of adjacent parts of the heart.

The development of a left ventricular aneurysm or even of an extensive dead area of myocardium after infarction greatly lowers the efficiency of the ventricle. ${ }^{5}$ The increased size of the cavity means that a greater tension is sustained in the myocardial wall for the same peak systolic ejecting pressure. As wall tension is a major determinant of the myocardium's metabolic need, 6 it may render an already critical coronary blood supply even more inadequate, with consequent worsening of heart failure and angina. Failure of an aneurysm to discharge its contents may be followed by thrombosis of blood within it and consequent embolization.

The incidence of ventricular aneurysm at postmortem examination of patients who had had a myocardial infarction has been found to be $3.5 \%,{ }^{7}$ but its precise diagnosis in life has been limited by the need to examine patients by contrast angiocardiography. An aneurysm should be suspected if left ventricular failure persists after myocardial infarction and particularly if it follows a first infarct. An electrocardiogram is usually helpful, because in most cases of aneurysm it shows persistent elevation of the ST segment in leads over the infarct. This is a well-established empirical observation, though its cause is not yet understood. $90 \%$ of aneurysms are anterolateral or apical ${ }^{8}$ and usually result from occlusion of the anterior descending branch of the left coronary artery; ${ }^{9}$ less often they protrude solely inferiorly or posteriorly. This common place for aneurysms means that they often show up on the plain radiograph of the chest, but, when the aneurysm is complicated by general left ventricular dilatation, any characteristic local deformation of the left ventricular border is likely to become obscured. Inferior aneurysms may sometimes be seen through the stomach gas bubble if they deform the diaphragm, and the more posterior ones often indent the oseophagus to become visible on a barium swallow. ${ }^{10}$ These are less commonly seen because of the likelihood of concomitant damage to the papillary muscles of the mitral valve, a particularly lethal complication.

To confirm the diagnosis the usual procedure is left ventricular cine angiography. Direct visualization of the contracting cavity in both oblique views enables the existence of an aneurysm to be confirmed or refuted with certainty, and it can be distinguished from generalized myocardial failure. Selective coronary angiography carried out at the same time 